\title{
THE APPROXIMATE NUMERICAL SOLUTION OF THE NON-HOMOGENEOUS LINEAR FREDHOLM INTEGRAL EQUATION BY RELAXATION METHODS*
}

\author{
BY \\ F. S. SHAW \\ Brown University
}

Summary. Relaxation methods are applied to the problem of finding an approximate numerical solution to the non-homogeneous linear Fredholm integral equation. As an illustration of the technique the deflection of a simply supported single span beam subjected to both normal and end loads is found.

1. The approximation to the integral equation. Consider the equation

$$
\varphi(x)=f(x)+\lambda \int_{a}^{b} K(x, t) \varphi(t) d t
$$

$\varphi(x)$ being the unknown function.

Let the interval $[a, b]$, of length $L$, be subdivided equally into $n$ parts as in Fig. 1., so that each subinterval is of length $L / n=l$ say.

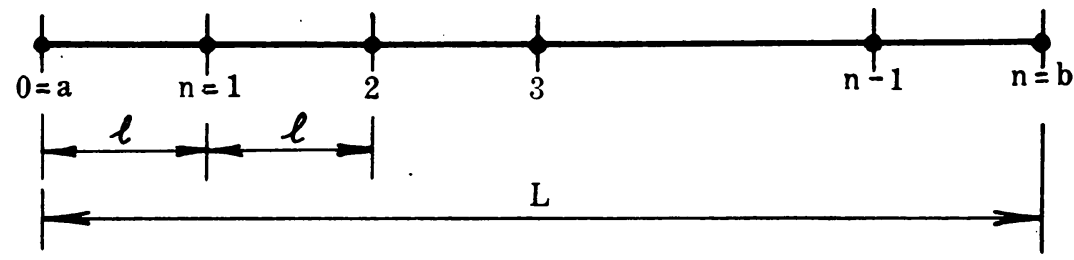

Fig. 1.

Then equation (1) may be replaced by the approximation

$$
\varphi(h) \approx f(h)+\lambda l\left[\frac{1}{2}\{K(h, a) \varphi(a)+K(h, b) \varphi(b)\}+\sum_{j=1}^{n-1} K(h, j) \varphi(j)\right],
$$

where $\varphi(h)$ represents the value of $\varphi(x)$ at the point $x=h, h=a, 1,2, \cdots, b$, and the integral $\int_{h}^{h+1} A(x) d x$ has been replaced by the approximation $\frac{1}{2} l(A(h)+A(h+1))$. To obtain an approximate solution to equation (1) it is necessary, then, to solve the set of $n+1$ simultaneous equations (2). This may conveniently be done by using the Relaxation technique.**

2. The residual and the relaxation operators. As a preliminary to solving, approximately, equations (2) it is necessary to construct two operators which, in the language of relaxation methods, are known as the residual operator and the relaxation operator respectively.

Let it be supposed that by means of an inspired guess (or by some other means) approximate values have been obtained for all the $\varphi(h)$. In general, the values will not be correct, i.e. will not satisfy equations (2), so that the error at each point is given by

\footnotetext{
*Received Dec. 17, 1947.

** Some knowledge of the technique is assumed, and it will not be discussed here. For further information see References 1, 2.
} 


$$
F(h)=f(h)+\lambda l\left[\frac{1}{2}\{K(h, a) \varphi(a)+K(h, b) \varphi(b)\}+\sum_{j=1}^{n-1} K(h, j) \varphi(j)\right]-\varphi(h),
$$

$F(h)$ being known as the residual at the point $h, h=a, 1, \cdots, n-1, b$.

Obviously, for an approximate solution of equations (2) we require that each $F(h)$ be (approximately) zero. To achieve this use is made of a relaxation operator.

Consider the effect of making an alteration of amount $\Delta \varphi(3)$ to the value of $\varphi(3)$ say. The resultant change in the residual at point 3 , i.e. $F(3)$, is given by

$$
\begin{aligned}
\Delta F(3) & =\frac{\partial F(3)}{\partial \varphi(3)} \Delta \varphi(3), \\
& =[\lambda l K(3,3)-1] \Delta \varphi(3)
\end{aligned}
$$

from equation (3). At the same time, and as a consequence of $\Delta \varphi(3)$, there is also a change in $F(2)$ of amount

$$
\begin{aligned}
\Delta F(2) & =\frac{\partial F(2)}{\partial \varphi(3)} \Delta \varphi(3) \\
& =\lambda l K(2,3) \Delta \varphi(3),
\end{aligned}
$$

with similar changes taking place in the residuals at the other $h$ stations.

More generally, the following effects result:

$$
\begin{aligned}
& \Delta F(h, j)=\left[\lambda l K(h, j)-\delta_{h i}\right] \Delta \varphi(j) \quad, \quad . \\
& \Delta F(h, a)=\frac{1}{2} \lambda l K(h, a) \Delta \varphi(a), \\
& \Delta F(h, b)=\frac{1}{2} \lambda l K(h, b) \Delta \varphi(b) \\
& \Delta F(a, a)=\left[\frac{1}{2} \lambda l K(a, a)-1\right] \Delta \varphi(a), \\
& \Delta F(a, h)=\lambda l K(a, h) \Delta \varphi(h) \quad,\} h, j=1,: 2, \cdots, n-1, \\
& \Delta F(a, b)=\frac{1}{2} \lambda l K(a, b) \Delta \varphi(b) \\
& \Delta F(b, b)=\left[\frac{1}{2} \lambda l K(b, b)-1\right] \Delta \varphi(b), \\
& \Delta F(b, h)=\lambda l K(b, h) \Delta \varphi(h) \\
& \Delta F(b, a)=\frac{1}{2} \lambda l K(b, a) \Delta \varphi(a)
\end{aligned}
$$

where $\Delta F(h, j)$ is the change in the residual at station $h$ resulting from a change in the value of $\varphi$, of amount $\Delta \varphi$, at station $j$. $\delta_{h j}$ is the kronecker delta*. In effect expressions (4) may be regarded as operators, and are known as the relaxation operators. For any arbitrary alteration to the value of the unknown function $\varphi$ at any one station

$$
{ }^{*} \delta_{h j}=\left\{\begin{array}{l}
1, \text { if } h=j, \\
0, \text { if } h \neq j
\end{array}\right.
$$


they give the resulting alterations to the errors in the solution at all stations. Thus they provide a means whereby the errors, or residuals, may be systematically reduced to as small a value as desired.

For actual use these operators are evaluated in tabular form. On putting $\Delta \varphi(h)=$ 1 , and dropping the $\Delta$ from $\Delta F(h, j)$ we have the unit operations Table I.

Table 1. Relaxation Operators

\begin{tabular}{|c|c|c|c|c|c|c|}
\hline \multirow{2}{*}{$\begin{array}{c}\Delta \varphi(h)=1, \\
\text { all the rest } \\
\text { zero. }\end{array}$} & \multicolumn{6}{|c|}{ Alteration to Residual $F(J)$. } \\
\hline & $F(a)$ & $F(1)$ & $F(2)$ & $\cdots$ & $F(n-1)$ & $F(b)$ \\
\hline$h=a$ & $\frac{1}{2} \lambda l K(a, a)-1$ & $\frac{1}{2} \lambda l K(1, a)$ & $\frac{1}{2} \lambda l K(2, a)$ & $\cdots$ & $\frac{1}{2} \lambda l K(n-1, a)$ & $\frac{1}{2} \lambda l K(b, a)$ \\
\hline$h=1$ & $\lambda l K(a, 1)$ & $\lambda l K(1,1)-1$ & $\lambda l K(2,1)$ & $\cdots$ & $\lambda l K(n-1,1)$ & $\lambda l K(b, 1)$ \\
\hline$h=2$ & $\lambda l K(a, 2)$ & $\lambda l K(1,2)$ & $\lambda l K(2,2)-1$ & $\cdots$ & $\lambda l K(n-1,2)$ & $\lambda l K(b, 2)$ \\
\hline$\cdots$ & $\cdots$ & $\cdots$ & $\cdots$ & $\cdots$ & $\cdots$ & $\cdots$ \\
\hline$h=n-1$ & $\lambda l K(a, n-1)$ & $\lambda l K(1, n-1)$ & $\lambda l K(2, n-1)$ & $\cdots$ & $\begin{array}{l}\lambda l K(n-1 \\
n-1)-1\end{array}$ & $\lambda l K(b, n-1)$ \\
\hline$h=b$ & $\lambda l K(a, b)$ & $\frac{1}{2} \lambda l K(1, b)$ & $\frac{1}{2} \lambda l K(2, b)$ & $\cdots$ & $\left|\frac{1}{2} \lambda l K(n-1, b)\right|$ & $\frac{1}{2} \lambda l K(b, b)-1$ \\
\hline
\end{tabular}

3. An example. In order to illustrate the method an example will be worked. Consider a single span beam simply supported at the ends and subjected to both normal and end loads. Both the normal loads and the moment of inertia of the beam may vary in any manner whatever. It is required to calculate the deflection of the beam.

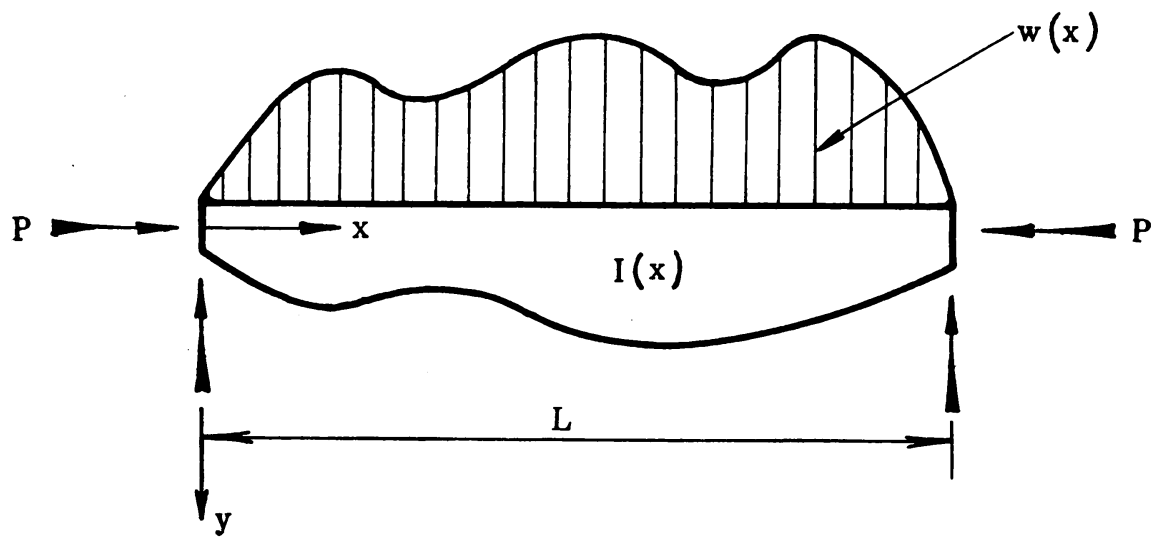

FIG. 2.

With the notation shown in Fig. 2., and using the Bernoulli-Euler theory of bending, the governing differential equation* is

*Fig. 2 is diagrammatic only. Actually it is assumed that the neutral plane of the beam is straight, that the beam cross section is symmetrical about a vertical axis, and that the force $P$ acts collinearly with the resulting straight line axis. 


$$
\frac{d^{2} y}{d x^{2}}=-\frac{M(x)}{E I(x)}
$$

which, for the simply supported beam shown, becomes

$$
\frac{d^{2} y}{d x^{2}}=-\frac{1}{E I(x)}\left(P y+M_{1}(x)\right) \text {. }
$$

$M_{1}(x)$ is the bending moment associated with the normal loading system $w\left(x_{x}\right)$, and so for any given $w(x)$ may be considered as known. $I(x)$ is the relevant moment of inertia of the beam cross section and may also be considered as known.

The integral equation appropriate to the problem is

$$
y(x)=\int_{0}^{L} K(x, \xi) A(\xi) M_{1}(\xi) d \xi+\int_{0}^{L} K(x, \xi) P A(\xi) y(\xi) d \xi,
$$

where $A(\xi)=1 / E I(\xi)$,

$$
K(x, \xi)=\left\{\begin{array}{l}
\xi(1-x / L) \text { for } 0 \leq \xi \leq x, \\
x(1-\xi / L) \text { for } x \leq \xi \leq L,
\end{array}\right.
$$

and $M_{1}(x)$ can be evaluated for each point $x$ once $w(x)$ is specified.

The integral equation is, therefore, of the form

$$
y(x)=f(x)+\lambda \int_{0}^{L} K(x, \xi) y(\xi) d \xi
$$

where $f(x), \lambda$ may be considered as known.

For comparison of accuracy it is convenient to choose a particular problem for which an exact solution is known. Accordingly it will be assumed that the load $w(x)$ is a uniformly distributed load, and that the moment of inertia is constant. For this particular case equation (6) becomes

$$
y(x)=\frac{w}{24 E I}\left(x^{4}+L^{3} x-2 L x^{3}\right)+\frac{P}{E I} \int_{0}^{L} K(x, \xi) y(\xi) d \xi,
$$

$K(x, \xi)$ being as given earlier.

As with most numerical procedures it is desirable to make the quantities concerned non-dimensional. To do this, put

$$
x=x_{1} L, \quad y=y_{1} L, \quad P L^{2} / E I=A, \quad w L^{3} / E I=B .
$$

On dropping the subscript 1 (for simplicity) equation (7) becomes

$$
y(x)=B \frac{x}{24}\left(x^{3}+1-2 x^{2}\right)+A \int_{0}^{1} K(x, \xi) y(\xi) d \xi, \quad 0 \leq x \leq 1 .
$$

For convenience* we choose $\lambda=A=2.5, B=0.5$, and take $n=10$ so that $l=1 / 10$. The relaxation operator table may then be completed without further discussion, the result being Table II.

*The exact value of the deflection at the center of the span is given by

$$
\frac{y}{L}=\frac{5}{384} \frac{w L^{3}}{E I} \frac{24\left(\sec u-1-\frac{1}{2} u^{2}\right)}{5 u^{4}},
$$

where $u=(L / 2)(P / E I)^{1 / 2}$. As $u \rightarrow 0$, sec $u \rightarrow 1$, as $u \rightarrow \pi / 2$, sec $u \rightarrow \infty$. Thus for $u=\pi / 4$ say, $P L^{2} / E I$ $=A=\pi^{2} / 4 \approx 2.5$. Also, with $y / L=(5 / 384) w L^{3} / E I \approx 1 / 150, B \approx 1 / 2$. 


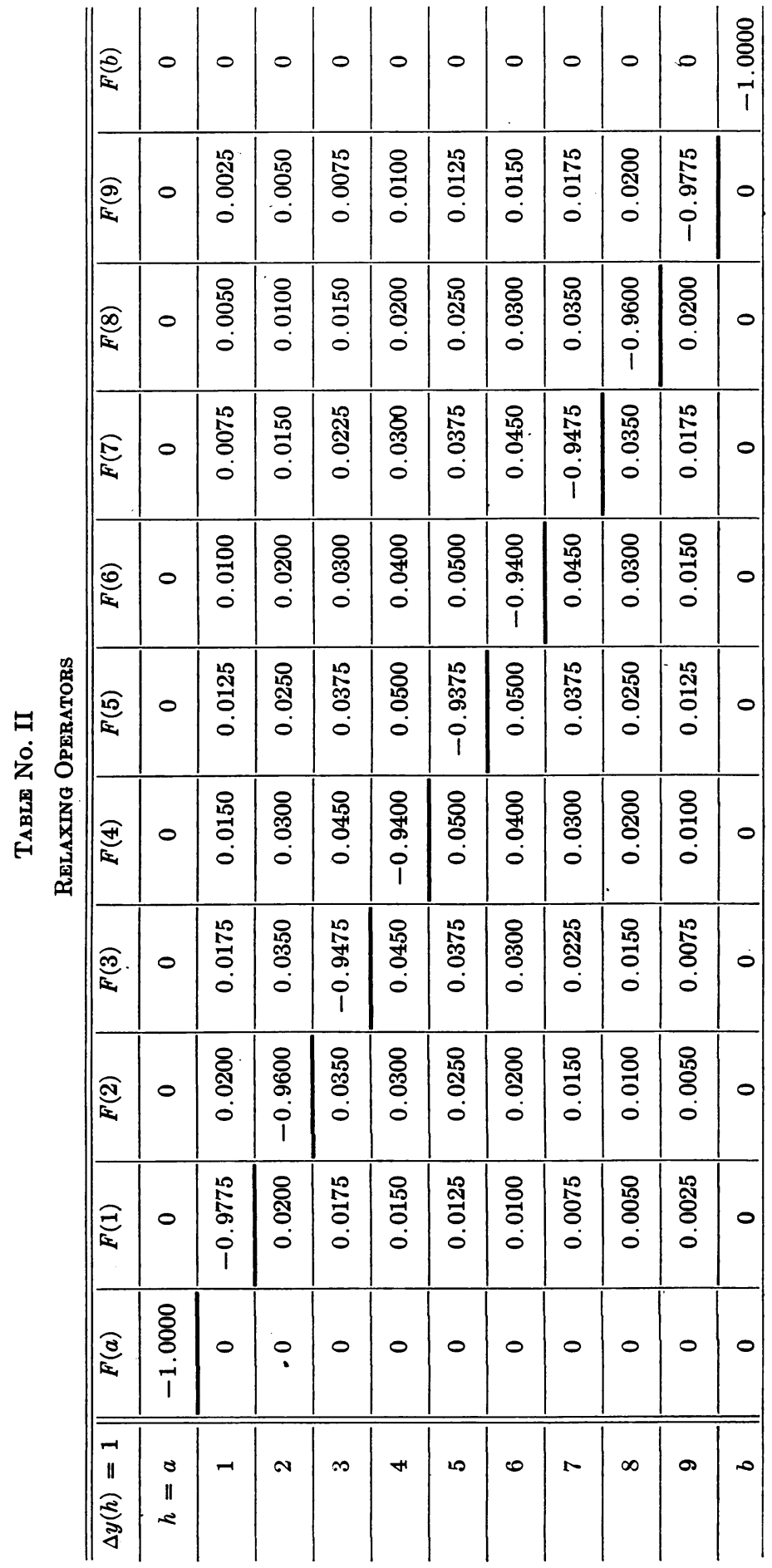


To compute the initial residuals, note that if the initial solution is taken to be $y=$ 0 everywhere, then $F(h)=f(h), h=a, 1, \cdots, 9, b$. This gives the first line in the relaxation table which follows-Table III. The relaxation process is then carried out in the usual manner. It will be seen that, since the operators are extremely powerful, convergence is very rapid.

From the nature of the problem solved here, and by inspection of the relaxation table, it is obvious that advantage could be taken of symmetry to cut down the work. It would merely have entailed combining operators 1 and 9, 2 and 8, etc., in Table II, and then only relaxing the residuals $F(a)$ to $F(5)$. For clarity however it was thought advisable not to do this.

4. Accuracy of solution. The exact value of the deflection is given by ${ }^{8}$

$$
\frac{y}{L}=\frac{w L^{3}}{16 E I u^{4}}\left[\frac{\cos u\left(1-2 x^{*} / L\right)}{\cos u}-1\right]-\frac{w L}{8 E I u^{2}} x(L-x),
$$

where $u=(L / 2)(P / E I)^{1 / 2}$.

Making this expression non-dimensional by use of the previous substitutions, and evaluating it for points $h=a, 1, \cdots, 5$, the following results are obtained:

\begin{tabular}{l||c|c|c|c|c|c}
\hline \hline & $h=a$ & 1 & 2 & 3 & 4 & 5 (center) \\
\hline Exact Solution. & 0 & $0.002,732$ & $0.005,175$ & $0.007,094$ & $0.008,308$ & $0.008,727$ \\
\hline Approximate Solution. & 0 & $0.002,737$ & $0.005,185$ & $0.007,106$ & $0.008,331$ & $0.008,751$ \\
\hline
\end{tabular}

The agreement is seen to be very good.

If greater accuracy were desired this could readily be obtained without increasing the number of subintervals by using a better approximation for the integration process. Thus, for example, Simpson's three point integration formula* could be used at the small expense of increasing slightly the initial labour necessary to compute the relaxation operators. However for some problems, depending on the terminal conditions, it might be desirable to decrease the lengths of the subintervals adjacent to the two ends $a$ and $b$.

It is evident that the method as given here may be applied equally well to obtain an approximate solution to the non-homogeneous linear Volterra integral equation.

\section{References}

1. R. V. Southwell, Relaxation methods in engineering science, Oxford Univ. Press, 1940.

2. F. S. Shaw, An introduction to relaxation methods (approximate methods of numerical computation), C. S. I. R. Div. of Aero. Report S M 78, Sept. 1946.

3. S. Timoshenko, Theory of elastic stability, McGraw-Hill Book Co., Inc., 1936. p. 8.

4. R. V. Southwell, Relaxation methods in theoretical physics, Oxford Univ. Press, 1946. p. 15.

*The use of more complicated integration formulae do not always lead to greater accuracy than that obtained by use of the simple trapezoidal rule. See, for example, ref. 4 . 


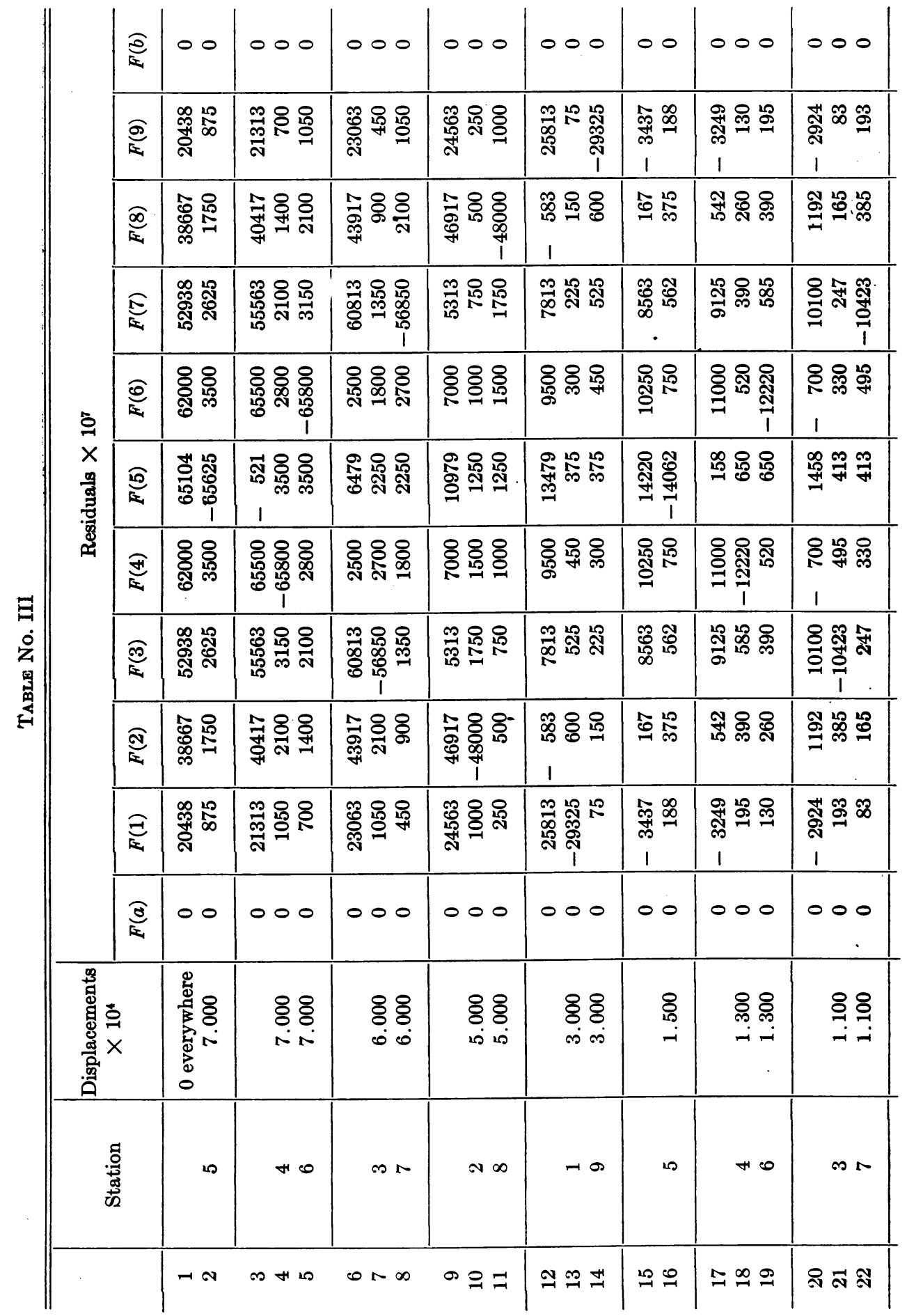


F. S. SHAW

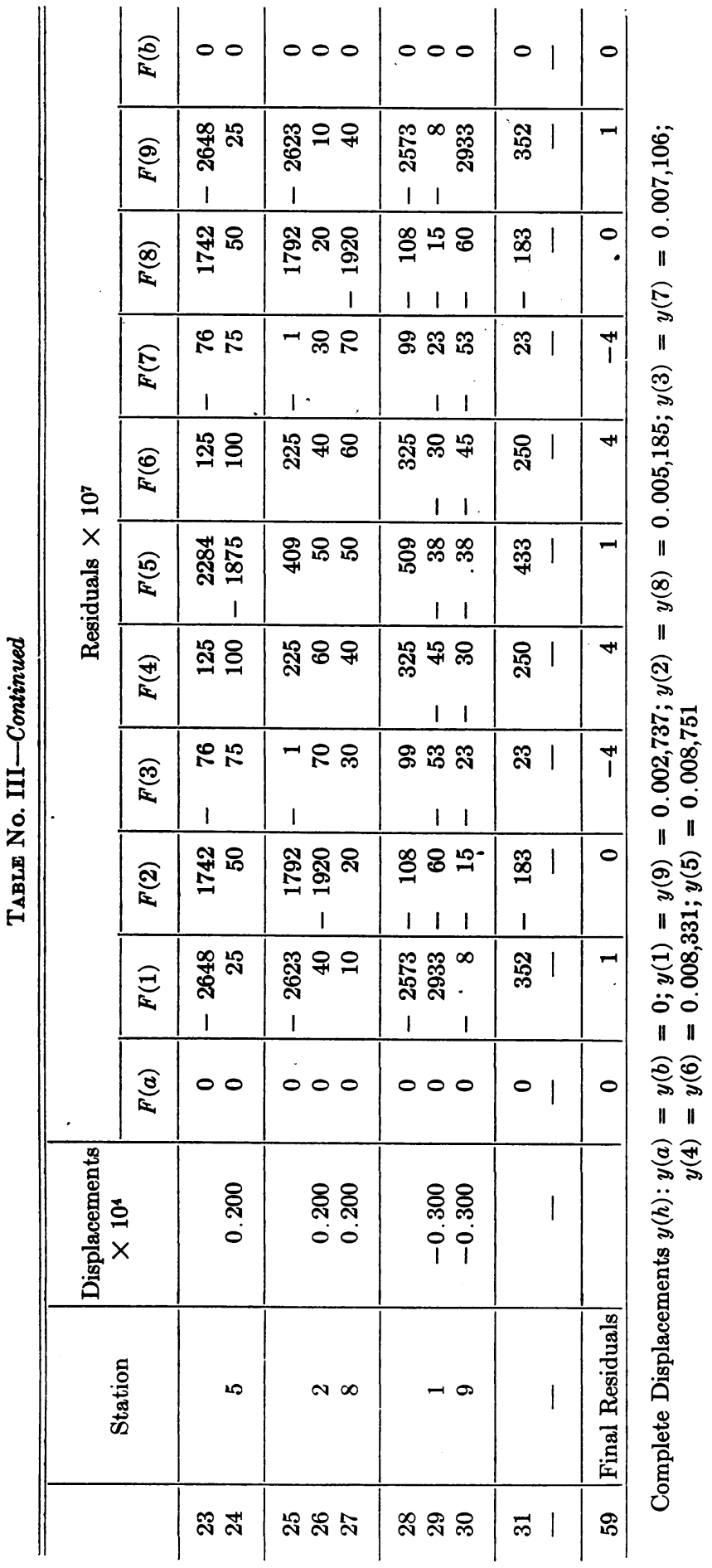

